\title{
ENDOGENOUS CHOICE ON ADVERTISING PRICING OF MEDIA PLATFORMS: LUMP-SUM FEE VS. PROPORTIONAL FEE*
}

\author{
LiJUN PAN \\ School of Economics, Nagoya University \\ Nagoya 464-8601, Japan \\ pan.lijun.nu@gmail.com
}

Received January 2016; Accepted September 2016

\begin{abstract}
Media platforms face the choice between lump-sum and proportional fees when they charge advertisers. This paper builds a two-stage dynamic game model to solve an endogenous choice problem with regard to the advertising pricing instruments of two media platforms. If either the proportional fee or the lump-sum fee is feasible, the dominant strategy for both platforms is to charge advertisers a proportional fee. This explains why online media platforms prefer to charge advertisers the proportional fee. We also examine the asymmetric pricing between two platforms that adopt different advertising schemes, which sheds light on the competition between online media and traditional media.
\end{abstract}

Keywords: media market, price instruments, two-sided platforms, endogenous choice, lumpsum fee, proportional fee

JEL Classification Codes: D43, L11, L13, L82

\section{Introduction}

Media platforms create value by connecting media consumers and advertisers. Examples include newspapers, magazines, and online news feeds. Although these media platforms usually generate revenues from consumers by subscription fees (lump-sum fee), they differ in their pricing schemes to advertisers, charging either a lump-sum or proportional fee. The online media platforms, such as online news feeds and search engines, charge advertisers based on the "cost-per-click" (CPC) rule or cost per thousand views (CPM), although these platforms can also charge advertisers a fixed amount of fee (lump-sum fee) (Evans, 2009). This gives rise to the following question: why do the online platforms prefer to charge advertisers a proportional fee when they can choose between a lump-sum fee and a proportional fee? On the other hand, unlike the online platforms, traditional media platforms, such as newspapers and magazines, find it difficult to adjust advertising fees in accordance to the fluctuating circulation because of

* I thank the co-editor, Motohiro Sato, an anonymous referee, Makoto Hanazono, Hikaru Ogawa, and Maria MartinRodriguez, for valuable comments and suggestions. The usual disclaimer applies. 
contract restrictions. Hence, they can only charge advertisers a lump-sum fee ${ }^{1}$. Since the online media platforms and the traditional media firms usually coexist in the same media market and face similar targeted consumers, how do these two types of media platforms that adopt different price instruments influence each other's prices and market shares? As empirically shown by Evans (2009), traditional media firms represented by newspapers are losing readers and advertisers to online media.

The above-mentioned questions motivate us to study how the pricing structure to advertisers affects the price competition among platforms, and to explore the platform's strategic choice of advertising fee. Specifically, this paper solves an endogenous choice problem with regard to the advertising pricing instruments of two platforms, provided that they both charge consumers a lump-sum fee. In the context of price competition with single-homing agents on both sides, we consider a two-stage game in which each platform decides whether to charge advertisers a lump-sum fee or a proportional fee in the first stage, and sets the prices to maximize their profits in the second stage. We find that in the subgame perfect Nash equilibrium, choosing to charge advertisers a proportional fee is the dominant strategy for both platforms. Hence, if both charging advertisers a proportional fee and a lump-sum fee are available to online platforms, they will choose the proportional fee. The economic intuition is as follows: switching from a lump-sum fee to a proportional fee affects both marginal and infra-marginal effects on the benefits from the consumers to the platform. On the one hand, the marginal benefit from an extra consumer is shared between the platform and the advertisers through the proportional fee. This implies that the benefit of an extra consumer on the marginal advertiser becomes lower, thereby reducing the number of new comers of advertiser. As a result, the marginal benefits from an additional consumer to the platform decline. On the other hand, this loss of marginal benefits is partly offset by a gain in the infra-marginal effect from the consumers to the platform. That is because the participation of an extra consumer enables the platform to extract from all of its advertisers an additional proportional fee. However, the loss of marginal effects outweigh the gain in the infra-marginal effects in equilibrium, so the total benefits brought by each consumer are reduced. This shows that, if they charge advertisers a proportional fee, the price competition between the platforms is weaker and the platforms make more profits. The above analysis explains the first question mentioned in the first paragraph.

Since choosing the proportional fee is always a dominant strategy for the media platform, when we consider the market where both the traditional media platforms and the online media platforms coexist, we can conclude that the online media platforms prefer to charge the advertisers the proportional fee. Nevertheless, to the traditional media platforms, only charging a lump-sum fee is available. To address the second question, we also investigate the asymmetric case when one platform (the traditional media platform) charges advertisers a lumpsum fee while the other (the online media platform) charges a proportional fee. We find that the online platform enjoys an advantage over the traditional platform in the market shares of both consumers and advertisers. This result theoretically verifies the empirical finding of Evans (2009). Furthermore, which platform charges each advertiser a higher price depends on the comparison of externalities. If the externality of consumers is higher than that of advertisers,

\footnotetext{
${ }^{1}$ Kaiser and Wright (2006) and Argentesi and Filistrucchi (2007) empirically find that magazines and newspapers charge advertisers a lump-sum fee.
} 
the rival platform will utilize its market share advantage of consumers to exploit advertisers with a higher price. These findings provide answers to the second question mentioned in the beginning.

The present paper relates to two strands of literature. First, it builds upon the works on the pricing behavior of firms in two-sided markets. Caillaud and Jullien (2003) analyze the case of non-differentiated platforms that provide pure intermediation service to homogenous users and charge both a lump-sum fee and a proportional fee, arguing that proportional fees act as a form of risk sharing between the platform and the agents. However, they do not consider the case when consumers are heterogeneous in their preferences, which is prevalent in the media market. Rochet and Tirole (2003) and Armstrong (2006) investigate the pricing behavior of two-sided platforms with heterogeneous consumers. The former focuses on the case of pure usage (proportional) fees and provide the classical argument on two-sided markets that one group's agents may subsidize the other group, while the latter places an emphasis on lump-sum fees. Following these two works, Gabszewicz et al. (2001), Crampes et al. (2009), and Reisinger (2011) assume that the advertisers are charged a lump-sum fee in their analysis on the media market, while Anderson and Coate (2005) and Peitz and Valletti (2008) assume that media platforms impose a proportional fee on advertisers. However, these works mainly assume one type of fee structure as exogenously given without looking into the effects of varying the price instruments. Rochet and Tirole (2006) build a canonical monopoly model that considers both a lump-sum fee and a proportional fee and obtain that the optimal pricing obeys the Lerner formula in both pricing regimes. However, they do not introduce the competition among platforms. In general, the above literature provides pioneering analyses of the properties of twosided markets, but does not consider the impacts of different pricing structures in the context of oligopolistic competition and heterogeneous agents. The present paper contributes to the first strand of literature by filling this research gap.

Second, this paper is also linked to a few works that compare the impacts of different pricing regimes on two-sided media markets. Dietl et al. (2013) compare the profit and welfare effects of a lump-sum fee versus a proportional fee to advertisers based on the competition between an ad-free (one-sided) pay media platform and a free media platform with advertisements, showing that the free (pay) media platform generates higher (lower) profits when the free media platform adopts a lump-sum fee than when it charges a proportional fee to advertisers. However, they do not consider a more general framework in which both platforms are two-sided and charge both sides a fee. In general, they still take different price instruments as exogenously given and compare them separately. ${ }^{2}$ This paper extends this strand of literature by endogenizing the choices on advertising pricing, and our results provide a new perspective of understanding the pricing behaviors of media platform.

Complementary to the above literature, this paper investigates media platforms' endogenous choice on different price instruments in an oligopolistic market with heterogeneous agents. In particular, the present paper is related to the single-homing two-part tariff model in Armstrong (2006). Both Armstrong (2006) and the present paper share the framework of a single-homing duopoly, and both consider the endogenous choice over price instruments. However, this paper deviates from that paper in three respects. First, it differs with respect to

\footnotetext{
${ }^{2}$ There are also several studies on the pricing structure of payment card systems, such as Wright (2011)and BedreDefolie and Calvano (2013), etc.
} 
the adoption of price instruments. Armstrong (2006) assumes that both platforms charge a twopart tariff consisting of both lump-sum fees and proportional fees, while this paper considers a binary choice model in which platforms choose between a lump-sum fee and a proportional fee to advertisers and charge consumers a subscription (lump-sum) fee. Second, this paper is distinct in terms of the timing of the game. Armstrong (2006) provides a one-shot game in which platforms decide on the price instruments and prices simultaneously. However, media platforms may first choose the price instruments, which are more difficult to adjust, and then set the optimal prices, as considered in this paper. Third, Armstrong (2006) analyzes the symmetric case when both platforms adopt the same pricing structure, but we also discuss the asymmetric case when platforms adopt different price instruments here.

The rest of this paper proceeds as follows. In Section II, we establish the duopoly model with two single-homing groups. In Section III, we derive the optimal prices and profits of the platforms regarding different price instruments to advertisers in the second stage, and obtain the optimal decision on the pricing to advertisers in the first stage. In Section IV, we discuss the prices, market shares and profits when a platform charges advertisers a lump-sum fee because it is too costly to adopt proportional charge, while the rival platform charges advertisers a proportional fee. Section V concludes.

\section{The Model}

Suppose there are two groups of agents, consumers and advertisers, who are connected by two competing platforms, $A$ and $B$. Group 1 and group 2 represent consumers and advertisers, respectively. Each consumer and advertiser choose to register on a single platform. The assumption that both groups single-home is widely employed in existing literature on twosided markets, such as Belleflamme and Toulemonde (2009) and Kotsogiannis and Serfes (2010). The reasons for both groups to single-home include the strong differentiation of the platform and limited resources of the agents. For instance, several empirical works on the media market, such as Kaiser and Wright (2006) and Argentesi and Filistrucchi (2007), show that few firms advertise on more than one newspaper or magazine at the same time. ${ }^{3}$ Groups 1 and 2 obtain utilities of $u_{1}^{i}$ and $u_{2}^{i}$, respectively, if they join platform $i$, where $i=A, B$. The utility of one group consists of the intrinsic value of the platform and the external benefits brought by the other group. A consumer obtains an intrinsic benefit of $v_{1}$ by joining either platform, and an advertiser obtains an intrinsic value of $v_{2}$. In addition, both consumers and advertisers care about the number of agents in the other group on the same platform. $\alpha_{1}$ measures the benefit a consumer brings to each advertiser, and $\alpha_{2}$ measures the benefit a consumer enjoys from each advertiser on the same platform. Here we assume $\alpha_{1}$ and $\alpha_{2}$ are both positive to cover most cases in the media market. ${ }^{4}$ To ensure full market coverage, we

\footnotetext{
${ }^{3}$ For the case when consumers single-home and advertisers multi-home, see Armstrong (2006). He finds that the equilibrium consumer price and the platforms' profit are higher (lower) when both platforms charge advertisers a proportional fee than when a lump-sum fee is levied to advertisers if readers like (dislike) ads. Yet he only considers the symmetric case when both platforms adopt the same price instruments, but does not investigate the asymmetric case when the platforms adopt different price instruments.

${ }^{4}$ It is controversial over the sign of the advertisement's externality to the consumers in the media market. There may be positive externality for the informative function of advertisement, and negative externality may also exist because of
} 
assume $v_{1}$ and $v_{2}$ are sufficiently large so that all consumers and advertisers participate.

Following the Hotelling specification, we assume the agents of each group are of mass one, and are uniformly distributed along a unit interval of $[0,1]$. The two platforms are located at the two endpoints of the interval, with platform $A$ located at 0 and platform $B$ located at 1 . A group- $k$ agent incurs a disutility $t_{k}\left|x_{k}-l^{i}\right|$ if he or she chooses platform $i(i=A, B)$, where $t_{k}$ $(k=1,2)$ is the differentiation parameter for group $k$, and $x_{k}$ represents the type of the agent in group $k$. For simplicity and without loss of generality, we assume $t_{1}=t_{2}=1$. To provide sharper results, we focus on the strength of cross-group externality relative to the differentiation parameter. Varying the differentiation parameter does not add more insight, but complicates the mathematics tremendously.

The two platforms charge consumers (group 1) a subscription (lump-sum) fee, and face a choice between a lump-sum charge and a proportional charge to advertisers (group 2). Specifically, platform $i$ charges a subscription fee $p_{1}^{i}$ to each consumer, while collects from advertisers either a lump-sum fee $p_{2}^{i}$ or a rate $\gamma_{2}^{i}$ in proportion to the number of its consumers. If $n_{1}^{i}$ consumers and $n_{2}^{i}$ advertisers join platform $i$, the utilities of a consumer and an advertiser are respectively

$$
\begin{array}{ll}
u_{1}^{i}=v_{1}+\alpha_{2} n_{2}^{i}-p_{1}^{i}-t_{1}\left|x_{1}-l^{i}\right|, \\
u_{2}^{i}=\left\{\begin{array}{ll}
v_{2}+\alpha_{1} n_{1}^{i}-p_{2}^{i}-t_{2}\left|x_{2}-l^{i}\right|, & \text { lump }- \text { sum fee } \\
v_{2}+\left(\alpha_{1}-\gamma_{2}^{i}\right) n_{1}^{i}-t_{2} \mid x_{2}-l^{i},, & \text { proportional fee }
\end{array},\right.
\end{array}
$$

With the Hotelling specification, the numbers of consumers and advertisers on platform $i$ are respectively

$$
\begin{aligned}
& n_{1}^{i}=\frac{1}{2}+\frac{u_{1}^{i}-u_{1}^{j}}{2} . \\
& n_{2}^{i}=\frac{1}{2}+\frac{u_{2}^{i}-u_{2}^{j}}{2} .
\end{aligned}
$$

Substituting expressions (3) and (4) into expressions (1) and (2), and using $n_{1}^{i}+n_{1}^{j}=1$ and $n_{2}^{i}+n_{2}^{j}=1$, yields the market shares of platform $i$ in the consumer and advertiser groups:

$$
\begin{aligned}
& n_{1}^{i}= \frac{1}{2}+\frac{\alpha_{2}\left(2 n_{2}^{i}-1\right)+\left(p_{1}^{j}-p_{1}^{i}\right)}{2} . \\
& n_{2}^{i}= \begin{cases}\frac{1}{2}+\frac{\alpha_{1}\left(2 n_{1}^{i}-1\right)+\left(p_{2}^{j}-p_{2}^{i}\right)}{2}, & \text { regime I } \\
\frac{1}{2}+\frac{\left(2 \alpha_{1}-\gamma_{2}^{j}-\gamma_{2}^{i}\right) n_{1}^{i}-\alpha_{1}+\gamma_{2}^{j}}{2}, & \text { regime II } \\
\frac{1}{2}+\frac{\left(2 \alpha_{1}-\gamma_{2}^{j}\right) n_{1}^{i}+\gamma_{2}^{j}-p_{2}^{i}-\alpha_{1}}{2}, & \text { regime III } \\
\frac{1}{2}+\frac{\left(2 \alpha_{1}-\gamma_{2}^{j}\right) n_{1}^{j}+p_{2}^{i}-\alpha_{1}}{2}, & \text { regime IV }\end{cases}
\end{aligned}
$$

the annoyance. Here we concentrate on the case where the overall externality is positive, which is empirically supported by Kaiser and Wright (2006). 
where both platforms charge advertisers a lump-sum fee in regime $I$; both platforms charge advertisers a proportional fee in regime II; platform $i(j)$ charges advertisers a lump-sum (proportional) fee in regime III; and platform $i(j)$ charges advertisers a proportional (lump-sum fee) in regime $I V$.

Without loss of generality, suppose both platforms incur zero constant cost to both groups. The profit of platform $i$ is

$$
\pi^{i}=\left\{\begin{array}{ll}
p_{1}^{i} n_{1}^{i}+p_{2}^{i} n_{2}^{i}, & \text { lump }- \text { sum fee } \\
p_{1}^{i} n_{1}^{i}+\gamma_{2}^{i} n_{1}^{i} n_{2}^{i} . & \text { proportional fee }
\end{array} .\right.
$$

The two platforms behave in a Nash manner to maximize their profits. The game is comprised of two stages. In the first stage, the two platforms noncooperatively and simultaneously choose between lump-sum fees and proportional fees to advertisers. In the second stage, they determine the price levels to maximize their profits, having observed each other's price instrument in the previous stage. We derive the adoption of price instruments in the subgame perfect Nash equilibrium by using backward induction.

\section{Competition in Price Instruments and Price Levels}

\section{Stage 2: Competition in Price Levels}

This section discusses the optimal prices under different pricing schemes. To clarify our discussion, we consider the following three regimes: (I) both platforms charge advertisers a lump-sum fee; (II) both platforms charge advertisers a proportional fee; (III) platform $A$ charges advertisers a lump-sum fee, whereas platform $B$ charges advertisers a proportional fee. Here we do not analyze the case when platform $B$ charges advertisers a lump-sum fee, while platform $A$ charges advertisers a proportional fee (regime $I V$ ), since such a situation is symmetric to regime $I I I$, and all the results in regime $I V$ can be easily derived from regime $I I I$. We make the following assumption to provide a meaningful solution:

Assumption: $0<\alpha_{i}<1, i=1,2$

This assumption provides a sufficient condition for the existence of a market-sharing equilibrium and positive prices in all regimes. ${ }^{5}$ (See Appendix A) It highlights the situation when differentiation is stronger than the cross-group externality, on which this paper concentrates.

Denote $p_{k M}^{i}$ as the lump-sum fee charged by platform $i(i=A, B)$ to group $k(k=1,2)$ in regime $M(M=I, I I, I I I)$, and $\gamma_{2 N}^{i}$ as the proportional fee charged by platform $i(i=A, B)$ to group 2 in regime $N(N=I I, I I I)$. The equilibrium prices of both platforms are expressed in the following lemma:

Lemma 1: In equilibrium, the price levels of the platforms with different price instruments are

\footnotetext{
${ }^{5}$ A sufficient and necessary condition for the existence of market sharing equilibrium is $1-\alpha_{1} \alpha_{2}>0$. However, this condition alone does not guarantee that all prices are positive in all regimes here. Therefore, we use Assumption 1 to guarantee that the market sharing equilibrium exists and all prices are positive.
} 
as follows:

(1-i) When both platforms charge advertisers a lump-sum fee (regime $I$ ),

$$
p_{1 I}^{i}=1-\alpha_{1} ; p_{2 I}^{i}=1-\alpha_{2}, i=A, B
$$

(1-ii) When both platforms charge advertisers a proportional fee (regime $I)$,

$$
p_{1 I I}^{i}=2-\alpha_{1}-\alpha_{2} ; \gamma_{2 I I}^{i}=2\left(1-\alpha_{2}\right), i=A, B
$$

(1-iii) When platform $A$ charges advertisers a lump-sum fee, while platform $B$ charges advertisers a proportional fee (regime $I I I$ ),

$p_{1 I I I}^{A}=\frac{1}{2 E}\left(2 \alpha_{1}^{4}-10 \alpha_{1}^{3}-11 \alpha_{1}^{2}+54 \alpha_{1}+8 \alpha_{2}^{4}-6 \alpha_{2}^{3}-13 \alpha_{2}^{2}+18 \alpha_{2}+26 \alpha_{1}^{2} \alpha_{2}^{2}-31 \alpha_{1}^{2} \alpha_{2}-29 \alpha_{1} \alpha_{2}\right.$

$\left.+24 \alpha_{1} \alpha_{2}^{3}-25 \alpha_{1} \alpha_{2}^{2}+\alpha_{1}^{2} D+\alpha_{2}^{2} D+3 \alpha_{1} \alpha_{2} D-6 D\right)$;

$p_{2 I I I}^{A}=\frac{1}{2 E}\left(2 \alpha_{1}^{3}+10 \alpha_{1}^{2}-9 \alpha_{1}-20 \alpha_{2}^{3}+19 \alpha_{2}^{2}+63 \alpha_{2}-6 \alpha_{1}^{2} \alpha_{2}-30 \alpha_{1} \alpha_{2}^{2}+25 \alpha_{1} \alpha_{2}-\alpha_{2} D+\alpha_{1} D-54\right) ;$

$p_{1 I I I}^{B}=\frac{1}{2 E}\left(2 \alpha_{1}^{4}-10 \alpha_{1}^{3}-5 \alpha_{1}^{2}+54 \alpha_{1}+4 \alpha_{2}^{2}+9 \alpha_{2}+10 \alpha_{1}^{3} \alpha_{2}+8 \alpha_{1} \alpha_{2}^{3}+16 \alpha_{1}^{2} \alpha_{2}^{2}-31 \alpha_{1}^{2} \alpha_{2}-22 \alpha_{1} \alpha_{2}^{2}\right.$

$\left.-8 \alpha_{1} \alpha_{2}-3 D+\alpha_{1}^{2} D+2 \alpha_{1} \alpha_{2} D-27\right)$;

$\gamma_{2 I I I}^{B}=\frac{1}{3+\alpha_{1}+2 \alpha_{2}}\left(2 \alpha_{1}^{2}+2 \alpha_{1}+4 \alpha_{1} \alpha_{2}+\alpha_{2}+D-9\right)$,

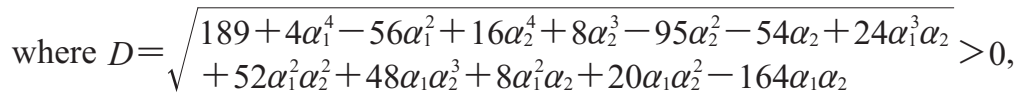

and $E=-27+5 \alpha_{1}^{2}+14 \alpha_{1} \alpha_{2}+8 \alpha_{2}^{2}<0$.

Proof: See Appendix A.

Q.E.D

Now we compare the prices in the three regimes. In order to make the prices to advertisers comparable, we denote $q_{2 I}^{i}=p_{2 I}^{i}$ and $q_{2 I I}^{i}=\gamma_{2 I I}^{i} n_{1 I I}^{i},(i=A, B), q_{2 I I I}^{A}=p_{2 I I I}^{A}$, and $q_{2 I I I}^{B}=\gamma_{2 I I}^{B} n_{1 I I I}^{B}$. We summarize the comparison of the price levels of different pricing schemes in the following proposition:

Proposition 1: (i) To consumers, both platform $A$ and platform $B$ charge the highest price in regime $I I$ and the lowest price in regime $I$, that is

$$
p_{1 I I}^{A}>p_{1 I I I}^{A}>p_{1 I}^{A} \text {; }
$$

(ii) To advertisers, platform $A$ charges a higher (lower) price, while platform $B$ charges a lower (higher) price, in regime III than in regimes I and II if $\alpha_{1}<\alpha_{2}\left(\alpha_{1}>\alpha_{2}\right)$, that is,

$$
\begin{aligned}
& \text { if } \alpha_{1}<\alpha_{2} \text {, then } q_{2 I I I}^{A}>q_{2 I}^{A}=q_{2 I I}^{A} \text { and } q_{2 I I I}^{B}<q_{2 I}^{B}=q_{2 I I I}^{B} \text {; } \\
& \text { if } \alpha_{1}>\alpha_{2} \text {, then } q_{2 I I I}^{A}<q_{2 I}^{A}=q_{2 I I}^{A} \text { and } q_{2 I I I}^{B}>q_{2 I}^{B}=q_{2 I I .}^{B} .
\end{aligned}
$$

\section{Proof: See Appendix B.}

To compare the prices intuitively, we first interpret the optimal prices in regimes $I$ and II. We transform the prices in Lemma (1-i) and (1-ii) into:

$$
p_{1 I}^{i}=1-\alpha_{1}\left(p_{2 I}^{i}+\alpha_{2}\right), p_{2 I}^{i}=1-\alpha_{2}\left(p_{1 I}^{i}+\alpha_{1}\right), i=A, B
$$




$$
\begin{gathered}
p_{1 I I}^{i}=1-\left(\alpha_{1}-\gamma_{2 I I}^{i}\right)\left(\frac{1}{2} \gamma_{2 I I}^{i}+\alpha_{2}\right)-\frac{1}{2} \gamma_{2 I I}^{i}, \\
\frac{1}{2} \gamma_{2 I I}^{i}=1-\alpha_{2}\left(p_{1 I I}^{i}+\alpha_{1}-\frac{1}{2} \gamma_{2 I I}^{i}\right), i=A, B
\end{gathered}
$$

Expressions (8) share the same features with Armstrong (2006). In a standard Hotelling model without cross-group externalities, the equilibrium price to group $j$ would be $p_{j I}=1$, i.e. the differentiation factor. With cross-group externality, the price is adjusted downward by $\alpha_{j}\left(p_{k l}+\alpha_{k}\right)$, which measures the marginal external benefit to a platform when an additional group $-j$ agent is attracted. First, an additional group- $j$ agent on the platform attracts $\alpha_{j}$ more group- $k$ agents, as shown in expressions (5) and (6). Second, the profit that the platform can extract from each of these extra group- $k$ agents is $\left(p_{k I}+\alpha_{k}\right)$, where $p_{k I}$ is the regular profit, and $\alpha_{k}$ is the extra profit the platform can gain from group- $j$ without losing market share. (When an extra group- $k$ agent joins a platform, the utility of a group- $j$ agent on that platform increases by $\alpha_{k}$, while the utility of a consumer on the rival platform decreases by $\alpha_{k}$. Hence the platform is able to increase the price to each group- $j$ agent by $2 \alpha_{k}$ without driving them away. Since the equilibrium number of group- $j$ agents is $\frac{1}{2}$, the extra revenue it can extract from its group- $j$ agents is $\alpha_{k}$.) Since $\alpha_{k}$ is the revenue that a platform can extract from each group- $k$ agent but is originally brought by an extra group- $k$ agent, it can be regarded as a feedback effect of group $k$.

Compared with the benchmark of expressions (8), expressions (9) shed some new light. In particular, the price to consumers differs in two respects. First, the price is adjusted downward by $\left(\alpha_{1}-\gamma_{2 I I}\right)\left(\frac{1}{2} \gamma_{2 I I}+\alpha_{2}\right)$. As shown in expressions (6), an additional consumer on the platform attracts $\alpha_{1}-\gamma_{2 I I}$ more group-2 agents. Because the platform is able to charge a price of $\gamma_{2 I I}$ when a consumer views or clicks on an advertisement, the net benefit from a consumer decreases by $\gamma_{2 I I}$. Hence the marginal attraction of advertisers falls by $\gamma_{2 I I}$ in comparison with regime $I$. In addition, similar to the interpretation in regime $I$, the profit that the platform can extract from each of these extra advertisers is $\frac{1}{2} \gamma_{2 I I}+\alpha_{2}$. $\left(\frac{1}{2} \gamma_{2 I I}\right.$ is the proportional fee $\gamma_{2 I I}$ multiplied by the equilibrium number of consumers $\frac{1}{2}$.) Hence the marginal effect of each consumer is reduced by $\gamma_{2 I I}\left(\frac{1}{2} \gamma_{2 I I}+\alpha_{2}\right)=2\left(1-\alpha_{2}\right)$

The second adjustment factor missing in regime $I$ is $n_{2 I I} \gamma_{2 I I}$. An extra consumer brings not only marginal external benefits, as explained earlier, but also infra-marginal external benefits to the platform. From the utility function of group-2 agents, an additional consumer brings a utility of $\alpha_{1}$ to each advertiser, from which the platform is able to extract $\gamma_{2 I I}$. Since the equilibrium number of advertisers present on the platform is $\frac{1}{2}$, the platform can extract a total profit of $n_{2 I I} \gamma_{2 I I}=\frac{1}{2} \gamma_{2 I I}$ from the advertisers because of this additional consumer. Thus $n_{2 I I} \gamma_{2 I I}=1-\alpha_{2}$ represents the infra-marginal external benefit to a platform from an additional consumer. 
Now we compare whether the reduction of marginal benefit or the positive infra-marginal benefit is stronger. Subtracting the infra-marginal effect by the marginal effect yields the net effect, which is $-\gamma_{2 I I}\left(\frac{1}{2} \gamma_{2 I I}-\frac{1}{2}+\alpha_{2}\right)$. The net effect is negative when $\gamma_{2 I I}>1-2 \alpha_{2}$, which is always true in equilibrium. That means the reduction in the marginal benefit of each consumer is only partly offset by its infra-marginal benefit. To be precise, the total benefit brought by each consumer to the advertisers decreases by $1-\alpha_{2}$ in regime II. Therefore, the price to consumers is higher in regime II than in regime $I$, and the competition for consumers between the platforms is mitigated.

Compared with regime $I$, the feedback effect of advertisers in regime $I I$ is also more complicated. $q_{2 I I}^{i}$ is constituted by the differentiation factor and the marginal external benefit from an additional advertiser. The first factor is the same as the standard Hotelling model. The second factor is also the number of consumers attracted by an additional advertiser ( $\alpha_{2}$ from equations (5)) multiplied by the extra benefit from each of the attracted consumers $\left(p_{1 I I}^{i}+\alpha_{1}-\frac{1}{2} \gamma_{2 I I}^{i}\right)$. With an extra consumer, an advertiser on the platform enjoys a net utility gain of $\alpha_{1}-\gamma_{2 I I}^{i}$ because he or she is charged a proportional fee $\gamma_{2 I I}^{i}$, while the utility of each advertiser on the rival platform falls by $\alpha_{1}$. Thus, the platform is able to increase the price to all the advertisers by $2 \alpha_{1}-\gamma_{2 I I}^{i}$ without losing any market share. Taking the equilibrium number of advertisers into account, the extra revenue from the advertisers because of an additional consumer is $\frac{1}{2}\left(2 \alpha_{1}-\gamma_{2 I I}^{i}\right)=\alpha_{1}-\frac{1}{2} \gamma_{2 I I}^{i}$. Therefore, the total effect to group 2 in regime II is the same as that in regime $I$, and hence the prices to advertisers in the two regimes are equal.

The price to consumers in regime III is between the prices in regimes $I$ and $I I$ because the marginal benefit brought by a consumer in this regime is also in between. In addition, if the externality from a consumer is higher than that from an advertiser $\left(\alpha_{1}>\alpha_{2}\right)$, platform $B$ would extract more surplus from advertisers by charging a higher fee, while platform $A$ would adopt the opposite strategy. The similar intuition also applies to the opposite case when the externality from a consumer is lower than that from an advertiser $\left(\alpha_{1}<\alpha_{2}\right)$.

\section{Stage 1: Choice on Price Instruments}

This section derives the optimal choice on price instruments for the two platforms. $\pi_{M}^{i}$ $(M=I, I I, I I I)$ represents the profit of platform $i$ in regime $M$. Based on Lemma 1, we obtain the maximized profits of the platforms in each type of pricing structure, as summarized in the following lemma.

Lemma 2: The maximized profits under different pricing structures are as follows:

(2-i) When both platforms charge advertisers a lump-sum fee (regime I),

$$
\pi_{I}^{i}=1-\frac{1}{2} \alpha_{1}-\frac{1}{2} \alpha_{2}, i=A, B
$$

(2-ii) When both platforms charge advertisers a proportional fee (regime II),

$$
\pi_{I I}^{i}=\frac{3}{2}-\frac{1}{2} \alpha_{1}-\alpha_{2}, i=A, B
$$


Table 1. Comparison of Platform Profits with Different

Advertising Pricing Schemes

\begin{tabular}{llcc}
\hline & & \multicolumn{2}{c}{ Platform $\boldsymbol{B}$} \\
\cline { 2 - 4 } & & Lump-sum fee & Proportional fee \\
\hline \multirow{2}{*}{ Platform $\boldsymbol{A}$} & Lump-sum fee & $\left(\pi_{I}^{A}, \pi_{I}^{B}\right)$ & $\left(\pi_{I I I}^{A}, \pi_{I I)}^{B}\right)$ \\
\cline { 2 - 4 } & Proportional fee & $\left(\pi_{I V}^{A}, \pi_{I V}^{B}\right)$ & $\left(\pi_{I I}^{A}, \pi_{I I}^{B}\right)$ \\
\hline
\end{tabular}

(2-iii) When platform $A$ charges advertisers a lump-sum fee, and platform $B$ charges advertisers a proportional fee (regime III),

$$
\pi_{I I I}^{A}=p_{1 I I I}^{A} n_{1 I I I}^{A}+p_{2 I I I}^{A} n_{2 I I I}^{A}, \pi_{I I I}^{B}=p_{1 I I I}^{B} n_{1 I I I}^{B}+\gamma_{2 I I I}^{B} n_{1 I I I}^{B} n_{2 I I I}^{B}
$$

where $n_{k I I I}^{i}(i=A, B$ and $k=1,2)$ represents the number of group- $k$ agent on platform $i$ in regime III. (For a detailed result in terms of the exogenous parameters, please see Appendix C.)

Proof: Since both platforms gain an even market share of both groups in the symmetric equilibrium, we can easily derive the profits for case (2-i) and case (2-ii) by substituting the equilibrium prices obtained in lemma 1 and the equilibrium numbers of agents into expressions (7). The proof for the profits in case (2-iii) is in Appendix C.

Q.E.D

Table 1 shows the profit matrix in the first stage, in which $\pi_{m n}^{i}$ is given by (2-i) to (2-iii).

Now we summarize the result in the following proposition.

Proposition 2: (i) Charging advertisers a proportional fee is a dominant strategy; (ii) Platforms make strictly more profits when they charge advertisers a proportional fee $\left(\pi_{I I}^{i}>\pi_{I I}^{i}>\pi_{I}^{i}\right)$; (iii) The platform charging advertisers a proportional fee gains a strict profit advantage over the one charging advertisers a lump-sum fee $\left(\pi_{I I}^{A}<\pi_{I I}^{B}\right)$.

Proof: See Appendix D.

Q.E.D.

Proposition 2 reveals that it is always more profitable for either platform to adopt the proportional advertising price no matter what pricing structure the rival platform chooses. That is because the proportional advertising fee reduces the marginal benefit of consumers and subsequently weakens competition. By charging advertisers a proportional fee, the platform induces the rival to charge consumers a higher fee, whether the rival charges advertisers a lump-sum fee or a proportional fee, as shown in the first statement of Proposition 1. If the rival charges advertisers a lump-sum fee, the platform is able to encroach some of the rival's market share and make more profit. If the rival also charges advertisers a proportional fee, the platform shares both markets equally with the rival but is able to set a higher price to consumers and subsequently gains more profit. In addition, when platforms adopting different price instruments coexist, the platform charging advertisers a lump-sum fee makes less profit than the one imposing a proportional fee. 


\section{Discussion}

So far we have assumed that the platforms can freely switch between lump-sum fee and proportional fee. As indicated by Proposition 2, charging advertisers a proportional fee is always a dominant strategy for the media platform. However, it is not feasible for some media firms to adopt proportional advertising fee. Traditional media firms, such as newspapers and magazines, find it difficult to adjust advertising fees in accordance to the fluctuating circulation because of contract restrictions. At the same time, online media firms are able to charge advertisers proportionally on a cost-per-click or cost-per-thousand-view basis. Therefore, the coexistence of two types of platforms that adopt different price instruments prevails, and it is worth comparing the prices and market shares between these platforms in this asymmetric setting. (i.e., regime III: the (lump-sum fee, proportional fee) case in Table 1)

We establish the result in the following proposition.

Proposition 3 In regime III, platform $A$ charges a higher consumer fee than platform $B$ and a higher (lower) advertising fee if $\alpha_{1}<\alpha_{2}\left(\alpha_{1}>\alpha_{2}\right)$, but has lower market shares than platform $B$ in both markets. That is,

(i) $p_{1 I I I}^{A}>p_{1 I I I}^{B}$;

(ii) If $\alpha_{1}<\alpha_{2}$, then $q_{2 I I I}^{A}>q_{2 I I I}^{B}$; if $\alpha_{1}>\alpha_{2}$, then $q_{2 I I I}^{A}<q_{2 I I I}^{B}$;

(iii) $n_{1 I I I}^{A}<n_{1 I I I}^{B}$ and $n_{2 I I I}^{A}<n_{2 I I I .}^{B}$.

Proof: See Appendix E.

Q.E.D.

The intuition can be roughly explained as follows. The equilibrium (at stage 2) in regime III is characterized by the system of first order conditions:

$$
\begin{gathered}
\frac{\partial \pi^{A}}{\partial p_{1}^{A}}=n_{1}^{A}+p_{1}^{A} \frac{\partial n_{1}^{A}}{\partial p_{1}^{A}}+p_{2}^{A} \frac{\partial n_{2}^{A}}{\partial p_{1}^{A}}=0 . \\
\frac{\partial \pi^{B}}{\partial p_{1}^{B}}=n_{1}^{B}+p_{1}^{B} \frac{\partial n_{1}^{B}}{\partial p_{1}^{B}}+n_{1}^{B} \gamma_{2}^{B} \frac{\partial n_{2}^{B}}{\partial p_{1}^{B}}+n_{2}^{B} \gamma_{2}^{B} \frac{\partial n_{1}^{B}}{\partial p_{1}^{B}}=0 . \\
\frac{\partial \pi^{A}}{\partial p_{2}^{A}}=n_{2}^{A}+p_{1}^{A} \frac{\partial n_{1}^{A}}{\partial p_{2}^{A}}+p_{2}^{A} \frac{\partial n_{2}^{A}}{\partial p_{2}^{A}}=0 . \\
\frac{\partial \pi^{B}}{\partial \gamma_{2}^{B}}=n_{1}^{B} n_{2}^{B}+p_{1}^{B} \frac{\partial n_{1}^{B}}{\partial \gamma_{2}^{B}}+\gamma_{2}^{B} n_{1}^{B} \frac{\partial n_{2}^{B}}{\partial \gamma_{2}^{B}}+\gamma_{2}^{B} n_{2}^{B} \frac{\partial n_{1}^{B}}{\partial \gamma_{2}^{B}}=0 .
\end{gathered}
$$

Comparing equations (10) and (11), we observe an extra negative effect of a rise in $p_{1}^{B}$ on platform $B$ 's profit, as expressed in the last term of equation (11). This effect is the inframarginal effect on the revenue from advertisers due to the fall in the number of consumers. Therefore, accounting for this extra effect, platform $B$ sets a lower price to consumers than platform $A$.

The intuition for the platforms' prices to group $B$ is as follows. We first derive the partial derivatives: $\frac{\partial n_{1}^{A}}{\partial p_{2}^{A}}=-\frac{\alpha_{2}}{N}, \frac{\partial n_{2}^{A}}{\partial p_{2}^{A}}=-\frac{1}{N}, \frac{\partial n_{1}^{B}}{\partial \gamma_{2}^{B}}=-\frac{\alpha_{2}}{N} n_{1}^{B}$, and $\frac{\partial n_{2}^{B}}{\partial \gamma_{2}^{B}}=-\frac{1}{2 N}\left[\left(p_{1}^{A}-p_{1}^{B}\right)+1+\alpha_{2}\left(2 n_{2}^{B}\right.\right.$ 
-1)], where $N=2\left(1-\alpha_{1} \alpha_{2}\right)+\alpha_{2} \gamma_{2}^{B}>0$. Substituting these partial derivatives into equations (12) and (13) yields a clearer comparison of the factors that influence the two platforms' pricing decision:

$$
\begin{gathered}
n_{2}^{A}-p_{1}^{A} \frac{\alpha_{2}}{N}+p_{2}^{A} \frac{\partial n_{2}^{A}}{\partial p_{2}^{A}}=0 . \\
n_{2}^{B}-p_{1}^{B} \frac{\alpha_{2}}{N}+\gamma_{2}^{B} \frac{\partial n_{2}^{B}}{\partial \gamma_{2}^{B}}-\gamma_{2}^{B} n_{2}^{B} \frac{\alpha_{2}}{N}=0 .
\end{gathered}
$$

Comparing equations (14) and (15), we can observe that platform $B$ has to take one more effect into account. Raising $\gamma_{2}^{B}$ by a marginal unit, platform $B$ suffers from an extra inframarginal loss stemming from the reduction of consumers, as characterized by the last term in equation (13). If $\alpha_{2}$ is relatively large, this potential loss (gain) from a higher (lower) $\gamma_{2}^{B}$ is relatively strong, and platform $B$ consequently has more incentive to charge a lower price to advertisers. Precisely, platform $B$ would charge a lower price if $\alpha_{2}>\alpha_{1}$.

The intuition for the comparison of market shares is straightforward. If platform $B$ charges a lower price than platform $A$ in both markets, it must enjoy a larger share of consumers and advertisers. If platform $B$ charges advertisers a higher price than platform $A$, it would not raise the price too high to lose the market share of advertisers because this strategy would further exert a negative impact on consumers' demand.

As indicated by Proposition 3, when the two platforms adopt different price instruments, the platform charging a proportional advertising fee gains advantages in its market shares of both groups, while which platform charges advertisers a higher price depends on the comparison of the two groups' externalities. This finding provides some theoretical implications to the media market, which is embodied with the coexistence of the traditional media firms that charge advertisers a lump-sum fee and the online media firms that charge advertisers proportional fees. Traditional media firms, such as city newspapers and magazines, mostly charge advertisers a lump-sum fee due to the difficulty to adjust prices in accordance to the fluctuating number of readers timely, but online media firms are able to employ the cost-perclick technology to levy flexible fees. Due to this distinction of pricing strategies, online media platforms are able to lower their prices to the readers so as to gain more shares in both markets. In reality, the encroachment of online media to traditional media is evident (Evans, 2009). The different pricing structures between these two types of firms may provide part of the explanation. Moreover, if advertisers care more about the readers or the audience than the other way round $\left(\alpha_{1}>\alpha_{2}\right)$, the traditional media platforms have to charge advertisers a lower price than the online media ones, as indicated by the proposition.

\section{Conclusion}

In reality, different media platforms adopt different price instruments when they charge advertisers. Existing literature mostly assumes either a lump-sum fee or a proportional fee, but overlooks platforms' endogenous choice on these two price instruments. This paper compares the optimal prices and profits under these two price instruments and solves an endogenous choice problem with regard to the advertising pricing instruments of two platforms competing 
for single-homing consumers and advertisers. In the subgame perfect Nash equilibrium, the dominant strategy is to adopt a proportional advertising fee, which explains why online media platforms prefer to charge advertisers a proportional fee. This paper also sheds light on the price competition between traditional media and online media, showing that the platform adopting a proportional advertising fee enjoys an advantage over the platform adopting a lumpsum advertising fee in terms of both market shares and profits. Besides, in this case, which platform charges each advertiser a higher price depends on the comparison of externalities. If the externality of consumers is higher than that of advertisers, the rival platform will utilize its market share advantage of consumers to exploit advertisers with a higher price.

The present paper is restricted to the case when both groups single-home. However, in reality, some groups may multi-home. For instance, consumers may read news from more than one platform. Therefore, a possible extension is to investigate the endogenous choice of price instruments when at least one group multi-homes. Another possible extension is to investigate the case when the externality of one group is negative, which may be complementary to the analysis of this paper. In sum, the present paper is just attempt to cast light on the pricing instruments choices among media firm.

\section{APPENDIX}

\section{A: Proof of Lemma 1 and Assumption}

Proof of Lemma 1-(i):

Solving the simultaneous equations (3) yields the market shares in terms of prices:

$$
n_{1}^{i}=\frac{1}{2}+\frac{\left(p_{1}^{j}-p_{1}^{i}\right)+\alpha_{2}\left(p_{2}^{j}-p_{2}^{i}\right)}{2\left(1-\alpha_{1} \alpha_{2}\right)} ; n_{2}^{i}=\frac{1}{2}+\frac{\left(p_{2}^{j}-p_{2}^{i}\right)+\alpha_{1}\left(p_{1}^{j}-p_{1}^{i}\right)}{2\left(1-\alpha_{1} \alpha_{2}\right)}
$$

Platform $i$ decides on the prices to the two groups in order to maximize its profits:

$$
\underset{p i, p i}{\operatorname{Max}} \pi^{i}=p_{1}^{i} n_{1}^{i}+p_{2}^{i} n_{2}^{i}
$$

The sufficient and necessary condition for a market-sharing equilibrium to exist is the second order condition of the platform's profit should be negative, i.e. $J=\left(\begin{array}{ll}\pi_{11} & \pi_{12} \\ \pi_{21} & \pi_{22}\end{array}\right)$ should be negative definite, where $\pi_{m n}=\frac{\partial \frac{\partial \pi}{\partial p_{m}}}{\partial p_{n}}\left(p_{m}^{*}, p_{n}^{*}\right)$

Here $J=-\frac{1}{2} \frac{1}{1-\alpha_{1} \alpha_{2}}\left(\begin{array}{cc}2 & \left(\alpha_{1}+\alpha_{2}\right) \\ \left(\alpha_{1}+\alpha_{2}\right) & 2\end{array}\right)$

$J$ is negative definite if and only if:

$$
\begin{aligned}
& \text { a. } / J_{1} /=-\frac{1}{1-\alpha_{1} \alpha_{2}}<0, \\
& \text { b. } / J_{2} /=\frac{4-\left(\alpha_{1}+\alpha_{2}\right)^{2}}{4\left(1-\alpha_{1} \alpha_{2}\right)}>0 .
\end{aligned}
$$


To ensure that a market-sharing equilibrium exists, the sufficient and necessary condition is:

$$
4>\left(\alpha_{1}+\alpha_{2}\right)^{2},
$$

which ensures that:

$$
1-\alpha_{1} \alpha_{2}>0 .
$$

which implies that the differentiation parameters $(1,1)$ should be larger than the network externality parameters $\left(\alpha_{1}, \alpha_{2}\right)$.

For the case of a symmetric equilibrium, each platform offers the same set of prices at:

$$
p_{1}=1-\alpha_{1}, p_{2}=1-\alpha_{2} \text {. }
$$

Proof of Lemma 1-(ii):

Solving the simultaneous equations (3) yields the market shares in terms of prices:

$$
\begin{aligned}
& n_{1}^{i}=\frac{1}{2}+\frac{2\left(p_{1}^{j}-p_{1}^{i}\right)+\alpha_{2}\left(\gamma_{2}^{j}-\gamma_{2}^{i}\right)}{2 H}, \\
& n_{2}^{i}=\frac{1}{2}+\frac{\left(2 \alpha_{1}-\gamma_{2}^{i}-\gamma_{2}^{j}\right)\left(p_{1}^{j}-p_{1}^{i}\right)+\left(\gamma_{2}^{j}-\gamma_{2}^{i}\right)}{2 H} .
\end{aligned}
$$

where $H=2\left(1-\alpha_{1} \alpha_{2}\right)+\alpha_{2}\left(\gamma_{2}^{i}+\gamma_{2}^{j}\right)$.

Platform $i$ decides on the prices to the two groups in order to maximize its profits:

$$
\underset{p_{1}^{i}, \gamma_{2}^{i}}{\operatorname{Max}} \pi^{i}=p_{1}^{i} n_{1}^{i}+\gamma_{2}^{i} n_{1}^{i} n_{2}^{i}, i=A, B
$$

The sufficient and necessary condition for a market-sharing equilibrium to exist is the second order condition of the platform's profit should be negative, i.e. $J=\left(\begin{array}{ll}\pi_{11} & \pi_{12} \\ \pi_{21} & \pi_{22}\end{array}\right)$ should be negative definite, where $\pi_{m n}=\frac{\partial \frac{\partial \pi}{\partial p_{m}}}{\partial p_{n}}\left(p_{m}^{*}, p_{n}^{*}\right)$.

Here $J=\frac{1}{81-\alpha_{1} \alpha_{2}+2 \alpha_{2}\left(1-\alpha_{2}\right)}\left(\begin{array}{cc}-4 & 2\left(2 \gamma_{2}+2 \alpha_{2}-\alpha_{1}-1\right) \\ -2 \alpha_{2} & \alpha_{2}-1\end{array}\right)$.

$J$ is negative definite if and only if:
a. $/ J_{1} /=-\frac{1}{21-\alpha_{1} \alpha_{2}+2 \alpha_{2}\left(1-\alpha_{2}\right)}<0$,
b. $/ J_{2} /=\frac{1-\alpha_{1} \alpha_{2}+2 \alpha_{2}\left(1-\alpha_{2}\right)+2 \alpha_{2} \gamma_{2}}{16\left[1-\alpha_{1} \alpha_{2}+2 \alpha_{2}\left(1-\alpha_{2}\right)\right]^{2}}>0$.

To ensure the existence of a market-sharing equilibrium, the necessary and sufficient condition is:

$$
1-\alpha_{1} \alpha_{2}+2 \alpha_{2}\left(1-\alpha_{2}\right)>0 .
$$

Given the condition for the existence of a market-sharing equilibrium, we have:

$$
H=2\left(1-\alpha_{1} \alpha_{2}\right)+\alpha_{2}\left(\gamma_{2}^{i}+\gamma_{2}^{j}\right)=2\left[1-\alpha_{1} \alpha_{2}+2 \alpha_{2}\left(1-\alpha_{2}\right)\right]>0 .
$$

For the case of a symmetric equilibrium, each platform offers the same set of prices at: 


$$
p_{1}=2-\alpha_{1}-\alpha_{2}, \gamma_{2}=2\left(1-\alpha_{2}\right)
$$

Proof of Lemma 1-(iii)

Solving the simultaneous equations (3) yields the market shares in terms of prices:

$$
\begin{gathered}
n_{1}^{A}=\frac{1}{2}+\frac{2\left(p_{1}^{B}-p_{1}^{A}\right)+\alpha_{2}\left(\gamma_{2}^{B}-2 p_{2}^{A}\right)}{2 I}, \\
n_{2}^{A}=\frac{1}{2}+\frac{\left(2 \alpha_{1}-\gamma_{2}^{B}\right)\left(p_{1}^{B}-p_{1}^{A}\right)+\left(\gamma_{2}^{B}-2 p_{2}^{A}\right)}{2 I}, \\
n_{1}^{B}=\frac{1}{2}+\frac{2\left(p_{1}^{A}-p_{1}^{B}\right)+\alpha_{2}\left(2 p_{2}^{A}-\gamma_{2}^{B}\right)}{2 I}, \\
n_{2}^{B}=\frac{1}{2}+\frac{\left(2 \alpha_{1}-\gamma_{2}^{B}\right)\left(p_{1}^{A}-p_{1}^{B}\right)+\left(2 p_{2}^{A}-\gamma_{2}^{B}\right)}{2 I} .
\end{gathered}
$$

where $I=2\left(1-\alpha_{1} \alpha_{2}\right)+\alpha_{2} \gamma_{2}^{B}$.

Platforms $A$ and $B$ decide on the prices to the two groups in order to maximize their profits:

$$
\begin{gathered}
\underset{p_{1}^{A}, p_{2}^{A}}{\operatorname{Max}} \pi^{A}=p_{1}^{A} n_{1}^{A}+p_{2}^{A} n_{2}^{A}, \\
\underset{\operatorname{Max}_{1}^{B} \gamma_{2}^{B}}{p^{B}}=p_{1}^{B} n_{1}^{B}+\gamma_{2}^{B} n_{1}^{B} n_{2}^{B} .
\end{gathered}
$$

The equilibrium prices are characterized by:

$$
\frac{\partial \pi^{A}}{p_{1}^{A}}=0, \frac{\partial \pi^{A}}{p_{2}^{A}}=0, \frac{\partial \pi^{B}}{p_{1}^{B}}=0, \frac{\partial \pi^{B}}{\gamma_{2}^{B}}=0 .
$$

The sufficient and necessary condition for a market-sharing equilibrium to exist is the second order condition of the platforms' profit should be negative, i.e.

$J^{i}=\left(\begin{array}{ll}\pi_{11}^{i} & \pi_{12}^{i} \\ \pi_{21}^{i} & \pi_{22}^{i}\end{array}\right)$ should be negative definite, where $\pi_{m n}^{i}=\frac{\partial \frac{\partial \pi^{i}}{\partial p_{m}}}{\partial p_{n}}\left(p_{m}^{i}, p_{n}^{i}\right)(i=A, B)$.

For platform $A, J^{A}=\frac{1}{I}\left(\begin{array}{cc}-4 & -2 \alpha_{1}-2 \alpha_{2}+\gamma_{2}^{B} \\ -2 \alpha_{1}-2 \alpha_{2}+\gamma_{2}^{B} & -4\end{array}\right)$.

$J^{A}$ is negative definite if and only if:

a. $/ J_{1}^{A} /=-\frac{4}{I}<0$,

b. $/ J_{2}^{A} /=\frac{16-\left(2 \alpha_{1}+2 \alpha_{2}-\gamma_{2}^{B}\right)^{2}}{I^{2}}>0$.

which obtains:

$$
I=2\left(1-\alpha_{1} \alpha_{2}\right)+\alpha_{2} \gamma_{2}^{B}>0 .
$$

Due to the complication of $J^{B}$, we provide a sufficient condition. By substituting $N>0$ into $J^{B}$, we find that $J^{B}$ is negative definite.

Hence to ensure the existence of a market-sharing equilibrium, the sufficient condition is:

$$
I=2\left(1-\alpha_{1} \alpha_{2}\right)+\alpha_{2} \gamma_{2}^{B}>0 .
$$


The equilibrium prices are:

$$
\begin{aligned}
p_{1}^{A}= & \frac{1}{2 E}\left\{2 \alpha_{1}^{4}+2 \alpha_{1}^{3}\left(-5+6 \alpha_{2}\right)+\alpha_{1}^{2}\left(-11-31 \alpha_{2}+26 \alpha_{2}^{2}+D\right)+2\left(9 \alpha_{2}-3 \alpha_{2}^{3}+4 \alpha_{2}^{4}-3 D\right)\right. \\
& +\alpha_{2}^{2}(-13+D)+\left[\alpha_{1}\left(54-25 \alpha_{2}^{2}+24 \alpha_{2}^{3}+\alpha_{2}(-35+3 D)\right]\right\}, \\
p_{2}^{A}= & \frac{1}{2 E}\left[-54+2 \alpha_{1}^{3}+\alpha_{1}^{2}\left(10-6 \alpha_{2}\right)+63 \alpha_{2}+19 \alpha_{2}^{2}-20 \alpha_{2}^{3}-\alpha_{2} D+\alpha_{1}\left(-9+25 \alpha_{2}-30 \alpha_{2}^{2}+D\right)\right], \\
p_{1}^{B}= & \frac{1}{2 E}\left\{-27+2 \alpha_{1}^{4}+10 \alpha_{1}^{3}\left(-1+\alpha_{2}\right)+9 \alpha 2+4 \alpha_{2}^{2}-3 D+\alpha_{1}^{2}\left(-5-31 \alpha_{2}+16 \alpha_{2}^{2}+D\right)\right. \\
& \left.+2 \alpha_{1}\left[27-11 \alpha_{2}^{2}+4 \alpha_{2}^{3}+\alpha_{2}(-4+D)\right]\right\}, \\
\gamma_{2}^{B}= & \frac{1}{3+\alpha_{1}+2 \alpha_{2}}\left[-9+2 \alpha_{1}^{2}+\alpha_{2}+\alpha_{1}\left(2+4 \alpha_{2}\right)+D\right] .
\end{aligned}
$$

where $D=\sqrt{\begin{array}{l}189+4 \alpha_{1}^{4}-54 \alpha_{2}+24 \alpha_{1}^{3} \alpha_{2}-95 \alpha_{2}^{2}+8 \alpha_{2}^{3}+16 \alpha_{2}^{4} \\ +4 \alpha_{1} \alpha_{2}\left(-41+5 \alpha_{2}+12 \alpha_{2}^{2}\right)+\alpha_{1}^{2}\left(-56+8 \alpha_{2}+52 \alpha_{2}^{2}\right)\end{array}}>0$,

and $E=-27+5 \alpha_{1}^{2}+14 \alpha_{1} \alpha_{2}+8 \alpha_{2}^{2}<0$.

Substituting expressions (A-6) into expressions (A-5), we have the equilibrium market shares:

$$
\begin{gathered}
n_{1}^{A}=\frac{1}{2}+\frac{3 F}{4 E}, \\
n_{2}^{A}=\frac{1}{2}+\frac{F}{4 E}\left(\alpha_{1}+2 \alpha_{2}\right), \\
n_{1}^{B}=\frac{1}{2}-\frac{3 F}{4 E}, \\
n_{2}^{B}=\frac{1}{2}-\frac{F}{4 E}\left(\alpha_{1}+2 \alpha_{2}\right) .
\end{gathered}
$$

where $F=-9+2 \alpha_{1}^{2}-3 \alpha_{2}+6 \alpha_{1} \alpha_{2}+4 \alpha_{2}^{2}+D>0$.

Proof of Assumption:

With the assumption of $0<\alpha_{1}<1$ and $0<\alpha_{2}<1$, we first examine the signs of the prices.

In regime $1, p_{1}=1-\alpha_{1}>0$ and $p_{2}=1-\alpha_{2}>0$;

In regime $2, p_{1}=2-\alpha_{1}-\alpha_{2}>0$ and $\gamma_{2}=2\left(1-\alpha_{2}\right)>0$;

In regime 3 , all the prices are proved to be positive.

Now we examine whether this assumption is sufficient for a market-sharing equilibrium. Inequalities $(A-2),(A-5)$ and $(A-8)$ are reduced to: $1-\alpha_{1} \alpha_{2}>0,1-\alpha_{1} \alpha_{2}+2 \alpha_{2}\left(1-\alpha_{2}\right)>0$, and $I=2\left(1-\alpha_{1} \alpha_{2}\right)+\alpha_{2} \gamma_{2}^{B}>$ $2\left(1-\alpha_{1} \alpha_{2}\right)>0$.

\section{B: Proof of Proposition 1}

Proof of Proposition 1-(i):

Denote Platform $A$ 's fees to consumers in regimes I, II and III as $p_{I I}^{A}, p_{1 I I}^{A}$ and $p_{1 I I I}^{A}$, respectively; and denote platform $B$ 's fees to consumers in regimes I, II and III as $p_{1 I}^{B}, p_{1 I I}^{B}$ and $p_{1 I I I}^{B}$, respectively. Then we can compare the prices to Group 1 from expressions (A-3), (A-6) and (A-9). First, we compare $p_{11}^{A}, p_{1 I I}^{A}$ and $p_{1 I I I}^{A}$. Given the constraints that $0<\alpha_{1}<1$ and $0<\alpha_{2}<1$, as well as $D>0$ and $E<0$, we have:

$p_{1 I}^{A}-p_{1 I I}^{A}=\left(1-\alpha_{1}\right)-\left(2-\alpha_{1}-\alpha_{2}\right)=\alpha_{2}-1<0$, 


$$
\begin{aligned}
p_{1 I}^{A}-p_{1 I I I}^{A} & =-\frac{1}{2 E}\left(-6+\alpha_{1}^{2}+3 \alpha_{1} \alpha_{2}+2 \alpha_{2}^{2}\right)\left(-9+2 \alpha_{1}^{2}-3 \alpha_{2}+6 \alpha_{1} \alpha_{2}+4 \alpha_{2}^{2}+D\right)<0, \\
p_{1 I I}^{A}-p_{1 I I}^{A} & =-\frac{1}{2 E}\left[2 \alpha_{1}^{4}+8 \alpha_{2}^{4}+12 \alpha_{1}^{3} \alpha_{2}+26 \alpha_{1}^{2} \alpha_{2}^{2}+24 \alpha_{1} \alpha_{2}^{3}+10 \alpha_{2}^{3}+7 \alpha_{1}^{2} \alpha_{2}+19 \alpha_{1} \alpha_{2}^{2}-31 \alpha_{1}^{2}-58 \alpha_{2}^{2}\right. \\
& \left.-91 \alpha_{1} \alpha_{2}-36 \alpha_{2}+\left(\alpha_{1}^{2}+\alpha_{2}^{2}+3 \alpha_{1} \alpha_{2}-6\right) D+108\right]>0 .
\end{aligned}
$$

The three inequalities above yield $p_{1 I}^{A}<p_{1 I I I}^{A}<p_{1 I I}^{A}$.

Now we compare $p_{1 I}^{B}, p_{1 I I}^{B}$ and $p_{1 I I I}^{B}$ :

$$
\begin{aligned}
p_{1 I}^{B}-p_{1 I I}^{B} & =\left(1-\alpha_{1}\right)-\left(2-\alpha_{1}-\alpha_{2}\right)=\alpha_{2}-1<0, \\
p_{1 I}^{B}-p_{1 I I I}^{B} & =-\frac{1}{2 E}\left(-3+\alpha_{1}^{2}+2 \alpha_{1} \alpha_{2}\right)\left(-9+2 \alpha_{1}^{2}-3 \alpha_{2}+6 \alpha_{1} \alpha_{2}+4 \alpha_{2}^{2}+D\right)<0, \\
p_{1 I I}^{A}-p_{I I I I}^{A}= & -\frac{1}{2 E}\left[2 \alpha_{1}^{4}+10 \alpha_{1}^{3} \alpha_{2}+16 \alpha_{1}^{2} \alpha_{2}^{2}+8 \alpha_{1} \alpha_{2}^{3}+16 \alpha_{2}^{3}+7 \alpha_{1}^{2} \alpha_{2}+22 \alpha_{1} \alpha_{2}^{2}-25 \alpha_{1}^{2}-28 \alpha_{2}^{2}-64 \alpha_{1} \alpha_{2}\right. \\
& \left.-45 \alpha_{2}+\left(\alpha_{1}^{2}+2 \alpha_{1} \alpha_{2}-3\right) D+81\right]>0 ;
\end{aligned}
$$

The three inequalities above yield $p_{1}^{B I}<p_{1}^{B I I}<p_{1}^{B I I}$.

Proof of Proposition 1-(ii):

Denote platform $A$ 's fees to advertisers in regimes I, II and III as $q_{2 I}^{A}=p_{2 l}^{A}, q_{2 I I}^{A}\left(=n_{2 I l}^{A} \gamma_{2 I I}^{A}\right)$ and $q_{2 I I I}^{A}=p_{2 I I I}^{A}$, respectively; and denote platform $B$ 's fees to advertisers in regimes I, II and III as $q_{2 I}^{B}=p_{2 l}^{B}$, $q_{2 I I}^{B}\left(=n_{2 I}^{B} \gamma_{2 I I}^{B}\right)$ and $q_{2 I I I}^{B}\left(=n_{2 I I I}^{B} \gamma_{2 I I}^{B}\right)$, respectively.. Then we can compare the prices to Group 1 from expressions (A-3), (A-6) and (A-9). First, we compare $q_{2 I,}^{A}, q_{2 I I}^{A}$ and $q_{2 I I I}^{A}$. Given the constraints that $0<\alpha_{1}<1$ and $0<\alpha_{2}<1$, as well as $D>0$ and $E<0$, we have:

$q_{2 l}^{A}=q_{2 I I}^{A}=1-\alpha_{2}$,

$q_{2 l}^{A}-q_{2 I I I}^{A}=-\frac{1}{2 E}\left(\alpha_{1}-\alpha_{2}\right)\left(2 \alpha_{1}^{2}-3 \alpha_{2}+6 \alpha_{1} \alpha_{2}+4 \alpha_{2}^{2}+D-9\right)$, which is negative if $\alpha_{1}<\alpha_{2}$ and positive if $\alpha_{1}>$ $\alpha_{2}$.

Thus, $q_{2 I I I}^{A}$ is higher (lower) than $q_{2 l}^{A}$ and $q_{2 I I}^{A}$ if $\alpha_{1}<\alpha_{2}\left(\alpha_{1}>\alpha_{2}\right)$.

Now we compare $q_{2 I,}^{B}, q_{2 I I}^{B}$ and $q_{2 I I I}^{B}$ :

$q_{2 l}^{B}=q_{2 I I}^{B}=1-\alpha_{2}$,

$q_{2 l}^{B}-q_{2 I I I}^{B}=\frac{1}{2 E}\left(\alpha_{1}-\alpha_{2}\right)\left(2 \alpha_{1}^{2}-3 \alpha_{2}+6 \alpha_{1} \alpha_{2}+4 \alpha_{2}^{2}+D-9\right)$, which is positive if $\alpha_{1}<\alpha_{2}$ and negative if $\alpha_{1}>\alpha_{2} ;$

Thus, $q_{2 I I I}^{B}<(>) q_{2 I}^{B}$ and $q_{2 I I}^{B}$ if $\alpha_{1}<\alpha_{2}\left(\alpha_{1}>\alpha_{2}\right)$.

\section{C: Proof of Lemma 2}

Substituting the equilibrium prices and market shares into expressions (3) yields:

$$
\begin{aligned}
& \pi_{I I}^{A}=\frac{1}{8 E^{2}}\left\{2 \alpha_{1}^{4}+2 \alpha_{1}^{3}\left(-5+6 \alpha_{2}\right)+\alpha_{1}^{2}\left(-11-31 \alpha_{2}+26 \alpha_{2}^{2}+D\right)+2\left(9 \alpha_{2}-3 \alpha_{2}^{3}+4 \alpha_{2}^{4}-3 D\right)\right. \\
& \left.+\alpha_{2}^{2}(-13+D)+\alpha_{1}\left[54-25 \alpha_{2}^{2}+24 \alpha_{2}^{3}+\alpha_{2}(-35+3 D)\right]\right\}+\frac{1}{8 E^{2}}\left[2 E+F\left(\alpha_{1}+2 \alpha_{2}\right)\right][-54+ \\
& \left.2 \alpha_{1}^{3}+\alpha_{1}^{2}\left(10-6 \alpha_{2}\right)+63 \alpha_{2}+19 \alpha_{2}^{2}-20 \alpha_{2}^{3}-\alpha_{2} D+\alpha_{1}\left(-9+25 \alpha_{2}-30 \alpha_{2}^{2}+D\right)\right] \\
& \pi_{I I I}^{B}=\frac{1}{2 E}\left(\frac{1}{2}-\frac{3 F}{4 E}\right)\left\{-27+2 \alpha_{1}^{4}+10 \alpha_{1}^{3}\left(-1+\alpha_{2}\right)+9 \alpha_{2}+4 \alpha_{2}^{2}-3 D+\alpha_{1}^{2}\left(-5-31 \alpha_{2}+16 \alpha_{2}^{2}+D\right)\right.
\end{aligned}
$$


$\left.+2 \alpha_{1}\left[27-11 \alpha_{2}^{2}+4 \alpha_{2}^{3}+\alpha_{2}(-4+D)\right]+\frac{1}{2\left(3+\alpha_{1}+2 \alpha_{2}\right)}\left[-9+2 \alpha_{1}^{2}+\alpha_{2}+\alpha_{1}\left(2+4 \alpha_{2}\right)+D\right]\right\}$

\section{D: Proof of Proposition 2}

Proof of Proposition 2-(i):

Given the constraints that $0<\alpha_{1}<1$ and $0<\alpha_{2}<1$, as well as $D>0, E<0$ and $F>0$, we have:

$\pi_{I V}^{A}-\pi_{I}^{A}=\pi_{I I}^{B}-\pi_{I}^{A}=\frac{1}{2 E}\left(\frac{1}{2}-\frac{3 F}{4 E}\right)\left\{2 \alpha_{1}^{4}-10 \alpha_{1}^{3}-5 \alpha_{1}^{2}+54+4 \alpha_{2}^{2}+9 \alpha_{2}+10 \alpha_{1}^{3} \alpha_{2}-3 D\right.$

$+16 \alpha_{1}^{2} \alpha_{2}^{2}+8 \alpha_{1} \alpha_{2}^{3}-31 \alpha_{1}^{2} \alpha_{2}-22 \alpha_{1} \alpha_{2}^{2}-8 \alpha_{1} \alpha_{2}+\alpha_{1}\left(\alpha_{1}+2 \alpha_{2}\right) D-27+\frac{1}{2\left(3+\alpha_{1}+2 \alpha_{2}\right)}$

$\left.\left[2 \alpha_{1}^{2}+2 \alpha_{1}+\alpha_{2}+8 \alpha_{1} \alpha_{2}+D-9\right]\right\}-\frac{\left(2-\alpha_{1}-\alpha_{2}\right)}{2}>0$

$\pi_{I I}^{A}-\pi_{I I}^{A}=\frac{1}{8 E^{2}}\left[2 \alpha_{1}^{4}-11 \alpha_{1}^{2}-10 \alpha_{1}^{3}+54 \alpha_{1}+8 \alpha_{2}^{4}-6 \alpha_{2}^{3}-13 \alpha_{2}^{2}+18 \alpha_{2}+12 \alpha_{1}^{3} \alpha_{2}+26 \alpha_{1}^{2} \alpha_{2}^{2}-31 \alpha_{1}^{2} \alpha_{2}\right.$

$\left.+24 \alpha_{1} \alpha_{2}^{3}-25 \alpha_{1} \alpha_{2}^{2}-35 \alpha_{1} \alpha_{2}+\left(\alpha_{1}^{2}-6+\alpha_{2}^{2}+3 \alpha_{1} \alpha_{2}\right) D\right]+\frac{1}{8 E^{2}}\left[2 E+F\left(\alpha_{1}+2 \alpha_{2}\right)\right]\left[-54+2 \alpha_{1}^{3}+10 \alpha_{1}^{2}\right.$

$\left.-9 \alpha_{1}-20 \alpha_{2}^{3}+19 \alpha_{2}^{2}+63 \alpha_{2}-6 \alpha_{1}^{2} \alpha_{2}-30 \alpha_{1} \alpha_{2}^{2}+25 \alpha_{1} \alpha_{2}+\left(\alpha_{1}-\alpha_{2}\right) D\right]-\left(\frac{3}{2}-\frac{\alpha_{1}}{2}-\alpha_{2}\right)<0$

$\pi_{I I I}^{B}-\pi_{I}^{B}=\pi_{I I}^{B}-\pi_{I}^{A}>0 ; \pi_{I V}^{B}-\pi_{I I}^{A}=\pi_{I I}^{A}-\pi_{I I}^{A}<0$.

Proof of Proposition 2-(ii):

Given the constraints that $0<\alpha_{1}<1$ and $0<\alpha_{2}<1$, we have:

$$
\pi_{I I}^{i}-\pi_{I}^{i}=\left(\frac{3}{2}-\frac{\alpha_{1}}{2}-\alpha_{2}\right)-\left(1-\frac{\alpha_{1}}{2}-\frac{\alpha_{2}}{2}\right)=\frac{1-\alpha_{2}}{2}>0 .
$$

Proof of Proposition 2-(iii):

Given the constraints that $0<\alpha_{1}<1$ and $0<\alpha_{2}<1$, as well as $D>0$ and $E<0$, we have:

$\pi_{I I I}^{4}-\pi_{I I}^{B}=\frac{1}{4 E^{2}}\left[-2916+24 \alpha_{1}^{6}-20 \alpha_{1}^{5}-354 \alpha_{1}^{4}+198 \alpha_{1}^{3}+1755 \alpha_{1}^{2}-486 \alpha_{1}+32 \alpha_{2}^{6}+64 \alpha_{2}^{5}-498 \alpha_{2}^{4}\right.$

$-378 \alpha_{2}^{3}+2079 \alpha_{2}^{2}+729 \alpha_{2}+194 \alpha_{1}^{5} \alpha_{2}+618 \alpha_{1}^{4} \alpha_{2}^{2}+976 \alpha_{1}^{3} \alpha_{2}^{3}+784 \alpha_{1}^{2} \alpha_{2}^{4}+288 \alpha_{1} \alpha_{2}^{5}-108 \alpha_{1}^{4} \alpha_{2}$

$+84 \alpha_{1} \alpha_{2}^{4}-187 \alpha_{1}^{3} \alpha_{2}^{2}-76 \alpha_{1}^{2} \alpha_{2}^{3}-1851 \alpha_{1}^{3} \alpha_{2}-3294 \alpha_{1}^{2} \alpha_{2}^{2}-2265 \alpha_{1} \alpha_{2}^{3}+387 \alpha_{1}^{2} \alpha_{2}-207 \alpha_{1} \alpha_{2}^{2}+4428 \alpha_{1} \alpha_{2}$

$+\left(162+54 \alpha_{1}-93 \alpha_{1}^{2}-10 \alpha_{1}^{3}+12 \alpha_{1}^{4}+27 \alpha_{2}-228 \alpha_{1} \alpha_{2}-36 \alpha_{1}^{3} \alpha_{2}+61 \alpha_{1}^{3} \alpha_{2}-84 \alpha_{2}^{2}-33 \alpha_{1} \alpha_{2}^{2}+102 \alpha_{1}^{2} \alpha_{2}^{2}\right.$

$\left.\left.-2 \alpha_{2}^{3}+60 \alpha_{1} \alpha_{2}^{3}+8 \alpha_{2}^{4}\right) D\right]<0$

\section{E: Proof of Proposition 3}

Given the constraints that $0<\alpha_{1}<1$ and $0<\alpha_{2}<1$, we can obtain the following inequalities through simple calculation:

$$
\begin{aligned}
& p_{1 I I I}^{A}-p_{1 I I I}^{B}=\frac{1}{2 E}\left(-3+\alpha_{1} \alpha_{2}+2 \alpha_{2}^{2}\right)\left(2 \alpha_{1}^{2}-3 \alpha_{2}+6 \alpha_{1} \alpha_{2}+4 \alpha_{2}^{2}+D-9\right)>0, \\
& q_{2 I I I}^{A}-q_{2 I I I}^{B}=p_{2 I I I}^{A}-n_{2 I I I}^{B} \gamma_{2 I I}^{B}=\frac{1}{E}\left(\alpha_{1}-\alpha_{2}\right)\left(2 \alpha_{1}^{2}-3 \alpha_{2}+6 \alpha_{1} \alpha_{2}+4 \alpha_{2}^{2}+D-9\right) .
\end{aligned}
$$

which is positive if $\alpha_{1}<\alpha_{2}$ and negative if $\alpha_{1}>\alpha_{2}$; 
From expressions (A-10), $E<0, F>0$, and the constraints that $0<\alpha_{1}<1$ and $0<\alpha_{2}<1$, we have:

$n_{1}^{A}=\frac{1}{2}+\frac{3 F}{4 E}<\frac{1}{2}, n_{2}^{A}=\frac{1}{2}+\frac{F}{4 E}\left(\alpha_{1}+2 \alpha_{2}\right)<\frac{1}{2}, n_{1}^{B}=\frac{1}{2}-\frac{3 F}{4 E}>\frac{1}{2}$,

$n_{2}^{B}=\frac{1}{2}-\frac{F}{4 E}\left(\alpha_{1}+2 \alpha_{2}\right)>\frac{1}{2}$.

Therefore, $n_{1}^{A}<n_{1}^{B}$ and $n_{2}^{A}<n_{2}^{B}$.

\section{REFERENCES}

Anderson, S. and S. Coate (2005), "Market Provision of Broadcasting: A Welfare Analysis," Review of Economic Studies 72, pp.947-972.

Argentesi, E. and L. Filistrucchi (2007), "Estimating Market Power in a Two-Sided Market: The Case of Newspapers," Journal of Applied Econometrics 22, pp.1247-1266.

Armstrong, M. (2006), "Competition in Two-Sided Markets," RAND Journal of Economics 37, pp.668-691.

Bedre-Defolie, Ö. and E. Calvano (2010), "Pricing Payment Cards," Toulouse School of Economics and Princeton University, mimeo.

Belleflamme, P. and E. Toulemonde (2009), "Negative Intra-Side Externalities in Two-Sided Markets," International Economic Review 50, pp.245-272.

Caillaud, B. and B. Jullien (2003), "Chicken \& Egg: Competition among Intermediation Service Providers," RAND Journal of Economics 34, pp.309-328.

Chakravorti, S. and R. Roson (2006), "Platform Competition in Two-Sided Markets: The Case of Payment Networks," Review of Network Economics 5, pp.118-142.

Crampes, C., C. Haritchabalet and B. Jullien (2009), "Advertising, Competition and Entry in Media Industries," Journal of Industrial Economics 57, pp.7-31.

Dietl, H., M. Lang and P. Lin (2013), "Advertising Pricing Models in Media Markets: LumpSum Versus Per-Consumer Charges," Information Economics and Policy 25, pp.257-271.

Evans, D.S. (2009), "The Online Advertising Industry: Economics, Evolution, and Privacy," Journal of Economic Perspectives 23, pp.37-60.

Gabszewicz J., D. Laussel and N. Sonnac (2001), "Press Advertising and the Ascent of the 'Pensee Unique',' European Economic Review 45, pp.641-651.

Hossain, T., D. Minor and J. Morgan (2011), "Competing Matchmakers: an Experimental Analysis," Management Science 57, pp.1913-1925.

Kaiser, U. and J. Wright (2006), "Price Structure in Two-Sided Markets: Evidence from the Magazine Industry," International Journal of Industrial Organization 24, pp.1-28.

Kotsogiannis, C. and K. Serfes (2010), "Public Goods and Tax Competition in a Two-Sided Market," Journal of Public Economic Theory 12, pp.281-321.

Peitz, M. and T. Valletti (2008), "Content and Advertising in the Media: Pay-TV Versus Freeto-Air," International Journal of Industrial Organization 26, pp.949-965.

Reisinger, M. (2011), "Platform Competition for Advertisers and Users in Media Markets," International Journal of Industrial Organization 30, pp.243-252.

Rochet, J. and J. Tirole (2003), "Platform Competition in Two-Sided Markets," Journal of the European Economic Association 1, pp.990-1029. 
Rochet, J.-C. and J. Tirole (2006), “Two-Sided Markets: A Progress Report,” RAND Journal of Economics 37, pp.645-667. 